Non-uniform Li deposition during cycling of Li metal batteries leads to poor cyclability. Li deposition takes place at the solid electrolyte interphase (SEI), which is typically made up of multiple species resulting from reactions between $\mathrm{Li}$ and the electrolyte. These species strongly influence Li deposition; in particular, LiF-rich SEIs have recently emerged as a leading strategy to improve cyclability. However, the working mechanism of LiF-rich SEIs is puzzling, because $\mathrm{LiF}$ intrinsically cannot conduct $\mathrm{Li}^{+}$. Understanding the mechanism is further complicated by the formation of LiF SEIs as they can be either generated in situ using a fluorinated electrolyte or introduced intentionally via an ex situ synthetic route. Now, Betar M. Gallant and colleagues from the Massachusetts Institute of Technology compare the effect of ex situ and in situ formed SEIs on Li cycling and clarify the roles of $\mathrm{LiF}$.

The researchers find that during cycling of a Li electrode with an ex situ fabricated $\mathrm{LiF}$ SEI layer, the SEI impedes $\mathrm{Li}^{+}$transport in the initial cycle - as expected from its high ionic resistance - but its structure quickly breaks down, creating a less resistive path for the Li to deposit. However, the SEI breakage also means that metallic $\mathrm{Li}$ is exposed to the electrolyte, and therefore the performance of subsequent cycling depends on how well and how quickly a robust SEI can be restructured. The in situ formed SEI, on the other hand, has a two-layered structure with a thin crystalline $\mathrm{LiF}$ inner layer and a thick amorphous outer layer consisting of organic and polymeric species from electrolyte decomposition. While the breakage also occurs in the thin LiF layer during cycling, the elasticity of the outer layer helps to maintain the SEI structural stability. Furthermore, the fluorinated electrolyte passivates metallic Li by forming new LiF so that the SEI is repaired, leading to better cyclability.

\title{
Changjun Zhang
}

Published online: 19 February 2020 https://doi.org/10.1038/s41560-020-0568-y 